\title{
Multiplexed fluorescence mediated tomography with temporal and spectral data
}

Ying $\mathrm{Mu}$

Vivian Pera

Mark Niedre 


\title{
Multiplexed fluorescence mediated tomography with temporal and spectral data
}

\author{
Ying Mu, Vivian Pera, and Mark Niedre* \\ Northeastern University, Department of Electrical and Computer Engineering, 360 Huntington Avenue, Boston, Massachusetts 02115, \\ United States
}

\begin{abstract}
We recently developed an algorithm for multiplexed fluorescence tomographic imaging of at least four fluorophores concurrently in the red and near-infrared wavelength region by jointly using spectral and temporal data. We report the design of a fluorescence tomography instrument that acquires spectral and temporal data, and validate its use in tissue-mimicking phantoms with four embedded fluorescent targets with highly overlapped spectral signatures. Critically, this requires measurement or computation of extended fluorophore signature libraries, which capture the variability in the measured signal due to the unknown position of the targets in the media. We demonstrate that we can demix and tomographically image all four fluorophores with zero image cross-talk, and $1 \mathrm{~mm}$ or better spatial resolution. @ 2016 Society of Photo-Optical Instrumentation Engineers (SPIE) [DOI: 10.1117/1. JBO.21.10.105001]
\end{abstract}

Keywords: optical instrumentation; fluorescence; tomography; time-resolved imaging; diffuse optical imaging.

Paper 160473R received Jul. 6, 2016; accepted for publication Aug. 31, 2016; published online Oct. 4, 2016.

\section{Introduction}

Fluorescence mediated tomography (FMT) is an emerging imaging modality that allows three-dimensional imaging of fluorescent targets in optically diffusive biological media. ${ }^{1,2}$ In recent years, there have been significant advances in the development of red-shifted fluorescent protein variants suitable for deep tissue imaging, as well as targeted fluorescent probes to specific biomolecules such as cells surface receptors ${ }^{3-5}$ and enzymes. ${ }^{6-9}$ In principle, this raises the possibility of performing elegant experiments in which multiple fluorescent targets are imaged simultaneously in live animals. In preclinical cancer research studies this could allow, e.g., imaging of a bulk tumor volume over time, ${ }^{10,11}$ as well as changes in multiple cancerassociated molecules in response to new treatments. ${ }^{12,13}$

While common in fluorescence microscopy, ${ }^{14-16}$ this type of "multiplexed" imaging is extremely challenging in FMT for a number of reasons. Biological tissue is highly attenuating except at red or near-infrared (NIR) wavelengths, with an absorption minimum around $730 \mathrm{~nm}$. Combined with limitations in detector technology, the usable wavelength region in FMT is practically limited to between 650 and $850 \mathrm{~nm}$. Commonly used organic fluorophores (such as Alexafluor or Cyanine dyes) have broad emission spectra, so that only one or two well spectrally separated fluorophores can normally be imaged simultaneously in deep tissue using purely spectral data. ${ }^{17}$

To address this limitation, we and others have explored the idea of jointly using both fluorophore emission lifetime and spectral data in FMT. ${ }^{18-22}$ In particular, organic NIR fluorophores typically have lifetimes on the order of 0.5 to $2 \mathrm{~ns}$ (with emission lifetimes usually being shorter at longer wavelengths). Our previous studies ${ }^{23,24}$ suggested that joint use of spectral and temporal data provides more accurate demixing performance than either alone. We showed in silico that this allows concurrent

\footnotetext{
*Address all correspondence to: Mark Niedre, E-mail: mniedre@ece.neu.edu
}

tomographic imaging of at least four fluorophores in the narrow 650 - to $850-\mathrm{nm}$ wavelength range.

However, we also showed that use of temporal data in the tomographic demixing problem is not straightforward, because the measured time-resolved signal from a fluorescent target in tissue depends on both the distance from the laser source to the target, and from the target to the detector position. The position of the target is, by definition, not known a priori, and therefore the signal temporal shape is also not known. Many chemometric demixing approaches (e.g., least squares fitting) require accurate knowledge of these underlying spectra, and therefore cannot be used for this problem. On the other hand, so-called "blind" demixing algorithms (e.g., principal component analysis) appear to be suboptimal because they ignore substantial information about the fluorophore which should aid in demixing. We recently addressed this problem and demonstrated that the underlying uncertainty in the library (i.e., due to the unknown target position) could be addressed using an "extended library" that reflected multiple source-detector-target pathways and a nonnegative least-squares algorithm. ${ }^{23}$ Use of this extended library allowed us to estimate variations in the measured signal due to target position, spectral uncertainty, lifetime uncertainty, or shift due to wavelength-dependent optical properties.

In the present work, we first describe a new instrument suitable for small animal imaging capable of measuring spectral and temporal data in high-density tomographic data sets. Second, we perform the first experimental validation of our multiplexed imaging algorithm. ${ }^{23}$ We demonstrate that our instrument and algorithm are capable of tomographically imaging at least four fluorphores concurrently, with no cross-talk between channels, spatial imaging accuracy on the order of $1 \mathrm{~mm}$, and good agreement of the fluorophore concentration ratio. Moreover, we demonstrate that our extended library can be generated through experimental measurement or computationally with comparable 
accuracy, underscoring the potential use of this approach in a wide range of FMT experiments.

\section{Methods and Materials}

\subsection{Instrument Design}

A diagram and photograph of our instrument are shown in Figs. 1(a) and 1(b), respectively. We used a pulsed super-continuum laser (NKT Photonics) with $80-\mathrm{MHz}$ repetition rate, fitted with an infrared beam-splitting unit, so that the emitted light was broadband in the visible and NIR range from $\sim 550$ to $850 \mathrm{~nm}$. Depending on the measurement, one of two optical paths was used to illuminate the sample. In the first path, fluorescence excitation light was generated by filtering the laser output with a 680-nm shortpass and 655/50-nm bandpass filter (Chroma Technology Corp), as indicated by the red line in Fig. 1(a). In the second path, the unfiltered broadband NIR light was used to measure wavelength-dependent optical properties of the samples. The path could be selected with a set of broadband laser mirrors (Edmund Optics) on flip-mounts.

We used dual motorized rotation stages similar to the configuration in our previously reported time-resolved scanner. ${ }^{25}$ Samples were mounted on an inner rotation stage, and two detector fibers were mounted on an outer rotation stage, so that an arbitrary number of scanning angles could be generated. Light escaping the surface of the sample was first filtered with 700-nm longpass filters (Chroma) and collected by two 1-mm multimode optical fibers that were coupled into Spec-PML-16C detector units (Becker \& Hickl, Berlin, Germany). Each Spec-PML-16C is composed of a multiwavelength detection assembly combining a PML-16C (16-channel multianode PMT) and a $1 / 8 \mathrm{~m}$ polychromator. In combination, the PMT-array and grating were configured to cover a $200-\mathrm{nm}$ wavelength range

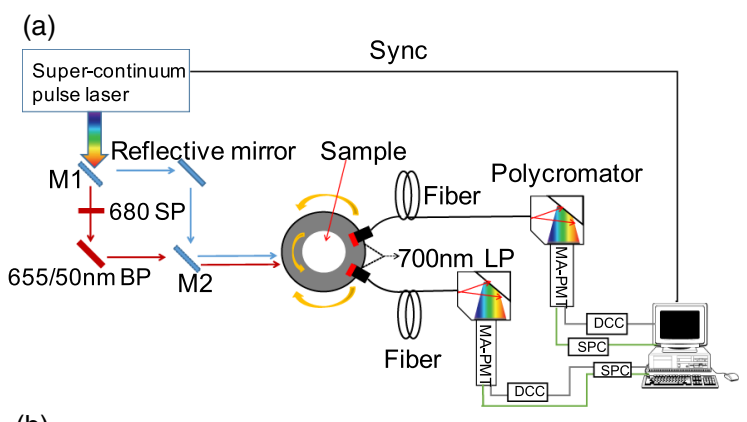

(i)

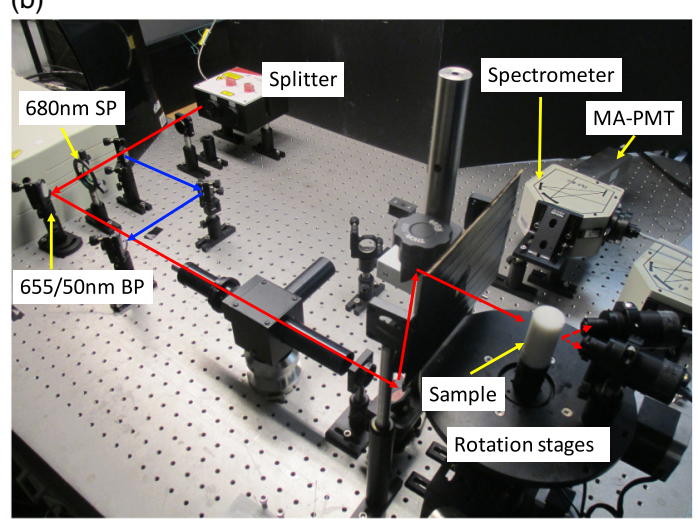

Fig. 1 (a) Diagram and (b) photograph of the time-resolved multiplexed FMT instrument. See text for component details. from 695 to $895 \mathrm{~nm}$. The detectors were controlled with a DCC card (Becker \& Hickl, Berlin, Germany) and connected to two SPC130 time-correlated single photon counting (TCSPC) modules (Becker \& Hickl), with 1024 time bins per measurement, covering $12.5 \mathrm{~ns}$ following the laser pulse. Data acquisition by the TCSPC cards was synchronized with the laser pulse by a trigger signal from the laser. Overall, this detection configuration allowed us to measure the emitted fluorescent signal with 12.7-ps temporal and 12.5-nm spectral resolution simultaneously.

\subsection{Optical Phantoms}

We characterized the instrument and algorithm with custom made optical phantoms. ${ }^{25}$ Briefly, the phantom is a hollowed out 25-mm diameter, 1-mm solid-walled cylinder, with 12, 1-mm diameter holes drilled in the base as shown in Figs. 2(a) and 2(b). This design allows us to flexibly insert tubes filled with fluorophore into the holes to generate an arbitrary number of spatial combinations. The hollow part of the phantom was filled with a liquid phantom to mimic the approximate optical
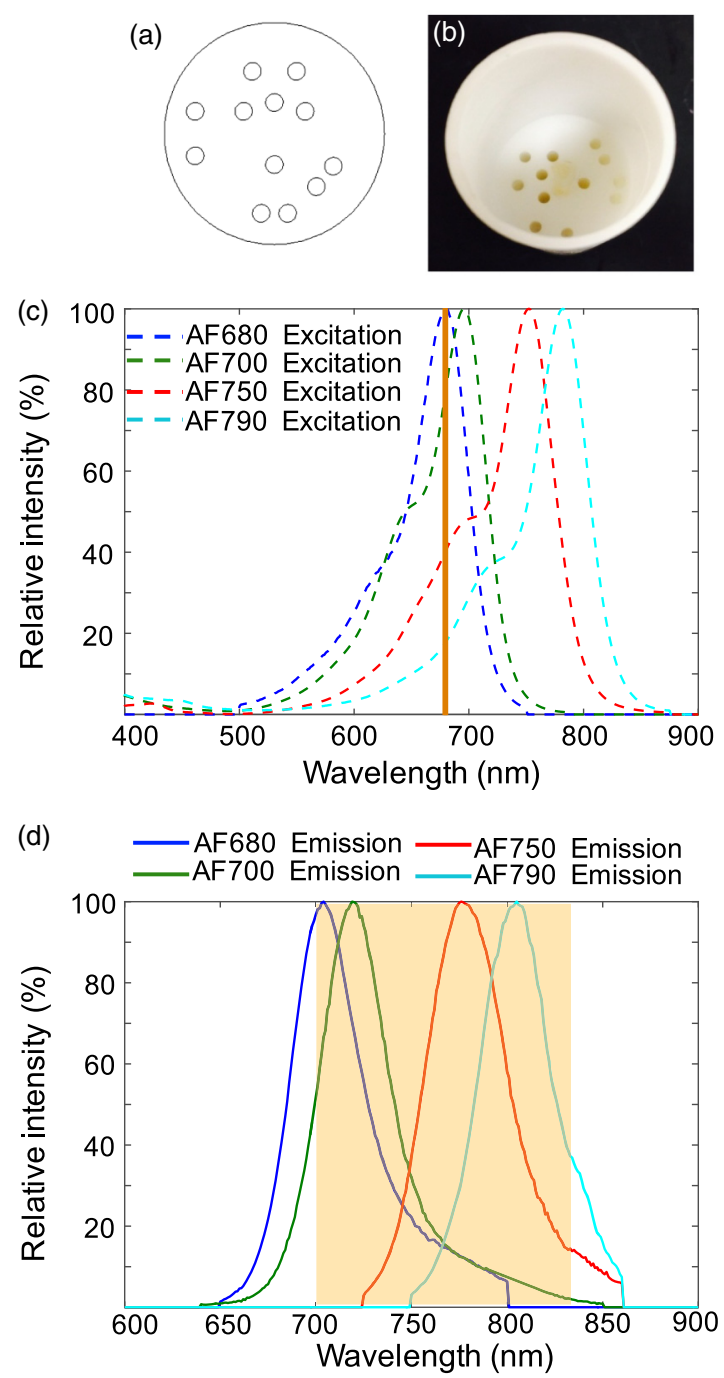

Fig. 2 (a) Diagram and (b) photograph of the optical phantom used in these experiments. Normalized (c) absorption and (d) emission spectra of Alexa Fluor 680, 700, 750, and 790. The vertical line in (c) and shaded rectangle in (d) represent the instrument excitation and collection wavelengths, respectively. 
Table 1 Photophysical properties of fluorophores used in these experiments.

\begin{tabular}{lccc} 
Fluorophore & $\begin{array}{c}\text { Excitation } \\
\text { maximum }\end{array}$ & $\begin{array}{c}\text { Emission } \\
\text { maximum }\end{array}$ & Lifetime \\
\hline Alexa Fluor 680 & $679 \mathrm{~nm}$ & $702 \mathrm{~nm}$ & $1.2 \mathrm{~ns}$ \\
Alexa Fluor 700 & $702 \mathrm{~nm}$ & $723 \mathrm{~nm}$ & $1.0 \mathrm{~ns}$ \\
Alexa Fluor 750 & $749 \mathrm{~nm}$ & $775 \mathrm{~nm}$ & $0.6 \mathrm{~ns}$ \\
Alexa Fluor 790 & $785 \mathrm{~nm}$ & $810 \mathrm{~nm}$ & $0.4 \mathrm{~ns}$ \\
\hline
\end{tabular}

properties of tissues. Specifically, we used $1 \%$ intralipid (SigmaAldrich, Michigan) with 50-ppm India ink added. Overall, the optical properties are $\sim \mu_{\mathrm{a}}=0.1 \mathrm{~cm}^{-1}, \mu_{\mathrm{s}}^{\prime}=10 \mathrm{~cm}^{-1}$ in the NIR region. ${ }^{26,27}$

We used our system to image several combinations of four different commonly used fluorophores: Alexa Fluor (AF) 680, AF 700, AF 750, and AF 790 (ThermoFisher Scientific). The normalized (to maximum) absorption and emission spectra for each are shown in Figs. 2(c) and 2(d). The vertical bar in Fig. 2(c) indicates the center emission wavelength of the laser in the first configuration (see Sec. 2.1); as indicated it allows us to excite all four of the fluorophores with varying efficiency due to the relative extinction coefficient for each. The shaded rectangle in Fig. 2(d) indicates the detection wavelength range of the spectrometers, indicating that our system is also capable of detecting the emission of all four fluorophores simultaneously. The absorption and emission maxima, as well as the fluorescence lifetime for each fluorophore are summarized in Table $1 .^{28}$

Three illustrative cases were studied for this paper:

1. AF680 $(1 \mu \mathrm{M})$ and AF700 $(1 \mu \mathrm{M})$, in two positions in the phantoms. The first at relatively superficial positions separated (center-to-center) by $11.5 \mathrm{~mm}$, and the second at deeper positions separated by $7 \mathrm{~mm}$.

2. AF680 $(1 \mu \mathrm{M})$, AF700 $(1 \mu \mathrm{M}), \operatorname{AF750}(1 \mu \mathrm{M})$, and A790 $(5 \mu \mathrm{M})$ in four different holes, separated by between 9.8 and $16.9 \mathrm{~mm}$.

3. To check for potential bleed between fluorophores, two sets of colocalized fluorphores in two holes, AF680 $(1 \mu \mathrm{M})$ with A750 $(1 \mu \mathrm{M})$ and AF700 $(1 \mu \mathrm{M})$ with AF790 $(5 \mu \mathrm{M})$.

During data acquisition, phantoms were placed on the inner rotation stage. The power of the laser light at the sample was
$37 \mathrm{~mW}$, and the gain of PMT detectors was set to 98\% (adjustable in Becker \& Hickl software). Phantoms rotated through $360 \mathrm{deg}$ with $5 \mathrm{deg}$ increments (72 angles). After each full rotation of the sample stage, the outer stage was rotated by $10 \mathrm{deg}$, and the acquisition was repeated. In total then, we positioned the detectors at six angular positions $(-45 \mathrm{deg},-25 \mathrm{deg}$, $-10 \mathrm{deg}, 0 \mathrm{deg},+35 \mathrm{deg}$, and $+45 \mathrm{deg}$ relative to the optical axis) and obtained $72 \times 6=432$ projective measurements. However, more or fewer measurements could easily be obtained by adjusting the rotation angles of the two stages. Each "measurement" here was 1024 temporal measurements (with 12.7-ps resolution) and 16-spectral measurements (with 12.5 -nm resolution), for a total of $7.1 \times 10^{6}$ data points per scan.

\subsection{Signal Demixing and Image Reconstruction}

We previously proposed and validated (in silico) a two-stage algorithm to efficiently utilize the large data sets generated by our system. ${ }^{23}$ This algorithm is described briefly here for completeness. The workflow is shown in Fig. 3. In the first stage, the measured temporal and spectral signal from each projection was demixed independently and accumulated tomographic measurements were extracted for each fluorophore using a signal library. In the second stage, an FMT reconstruction was performed to retrieve the location and concentration for each fluorophore.

\subsubsection{Fluorophore demixing}

In the demixing stage, we assume the measured signal is a linear combination of signals purely from each fluorophore.

$\mathbf{m}_{k}=\mathbf{S} \mathbf{a}_{k}+\mathbf{n}$,

with

$\mathbf{S}=\left[\begin{array}{llll}\mathbf{S}_{1} & \mathbf{S}_{2} & \cdots & \mathbf{S}_{f}\end{array}\right]$,

and

$\mathbf{a}_{k}=\left[\begin{array}{c}\mathbf{a}_{k, 1} \\ \mathbf{a}_{k, 2} \\ \vdots \\ \mathbf{a}_{k, f}\end{array}\right]$,

where $\mathbf{m}_{k}$ denotes the temporal-spectral data for the $k$ 'th measurement and is of size $T L \times 1 . T$ is the number of discrete time bins and $L$ is the number of wavelength channels (1024 and 16 in this case, respectively). Each column of $\mathbf{S}$ represents a temporal-spectral signature for a given fluorophore, which was generated using one of the two methods described in Sec. 2.4.

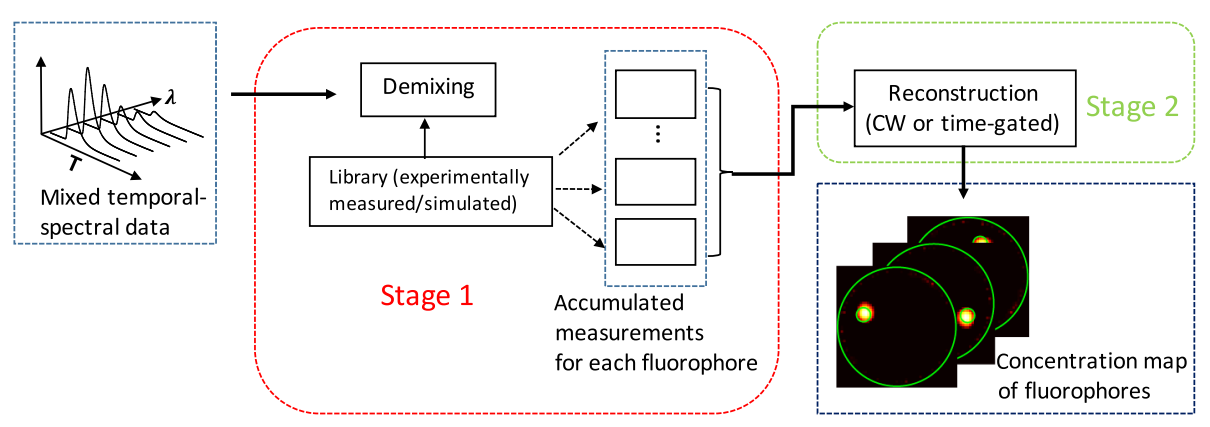

Fig. 3 Workflow of the two-stage demixing and reconstruction algorithm used in this study. 
The size of $\mathbf{S}_{f}$ is $T L \times N$, where $N$ refers to the number of signatures associated with each fluorophore. Importantly, multiple signatures for each fluorophore are required for the demixing problem in the case of tomography, i.e., to capture the variation in signal due to different light paths, due to relative positions of source, target, and detector which are unknown. Based on our previous results we used $N=3$ here, but this could be increased or decreased in the future. The vector $\mathbf{a}_{k}$ is a combining coefficient vector of dimension $F N \times 1$ for the $k$ 'th source-detector measurements, and $\mathbf{n}$ is an independent noise vector. Given $\mathbf{m}_{k}$ and $\mathbf{S}$, we solve Eq. (1) for $\mathbf{a}_{k}$ by solving a constrained minimization problem

$$
\underset{\mathbf{a}_{k} \geq 0}{\arg \min }\left\|\mathbf{m}_{k}-\mathbf{S a}_{k}\right\|_{2}^{2}
$$

We used MATLAB ${ }^{\circledR}$ "lsqnonneg” function (MathWorks, Natick, Massachusetts) which implements Lawson and Hanson's nonnegative least square algorithm. ${ }^{23}$ After $\mathbf{a}_{k}$ is obtained, the pure contribution from the $f$ 'th fluorophore is computed by using formula:

$$
\mathbf{m}_{k}^{(f)}=\mathbf{S}_{F} \mathbf{a}_{k, f}
$$

This process is repeated for every projective measurement. Thus we obtain the temporal-spectral data for all measurements of a single type of fluorophore:

$\mathbf{M}^{(f)}=\left[\mathbf{m}_{1}^{(f)} \mathbf{m}_{2}^{(f)} \cdots \mathbf{m}_{k}^{(f)} \cdots\right]$.

\subsubsection{Tomographic reconstruction}

In the second stage, we used the individual recovered (demixed) fluorophore measurement $\mathbf{M}^{(f)}$ obtained in the first stage and reconstructed the fluorophore's distribution in the phantom, one by one. Although in principle we could use the temporal properties of the signal (e.g., different time gates), the purpose of this work was to explore the demixing capabilities of the system as opposed to optimizing the reconstruction stage. Therefore, we summed the data over wavelength and time to generate quasicontinuous wave data sets. We used the standard linear formulation for a fluorescence field measured at the boundary for one source-detector pair using the diffusion approximation to the Boltzmann transport equation in an infinite medium to compute the Jacobian $(\mathrm{J})$. The tomography problem is then

$\mathbf{M}_{(\mathrm{cw})}^{(f)}=\mathbf{J} \eta(\mathbf{r})$,

where $\mathbf{M}_{(\mathrm{cw})}^{(f)}$ is the quasi-CW set of all fluorescence measurements, and $\eta(\mathbf{r})$ is the product of extinction coefficient, quantum yield of certain fluorophore type, and unknown concentration of that fluorophore at each position (r) in the medium, which was discretized into $0.5 \times 0.5 \mathrm{~mm}^{2}$ pixels. We solved Eq. (7) using the randomized algebraic reconstruction technique, with 400 iterations and relaxation parameter set to be $0.8 .^{29}$

\subsection{Generation of Extended Libraries}

To implement this demixing algorithm, it was necessary to generate an extended library of fluorophore signatures ' $\mathbf{S}$ ' [Eqs. (1) and (2), above] to capture the unknown source-target-detector pathlength variability in the tomographic problem. We used two methods to do this.

\subsubsection{Method 1: experimental measurement}

As shown in Fig. 4(a), a straw filled with each fluorophore at a concentration of $1 \mu \mathrm{M}$ was inserted into a fixed hole on the

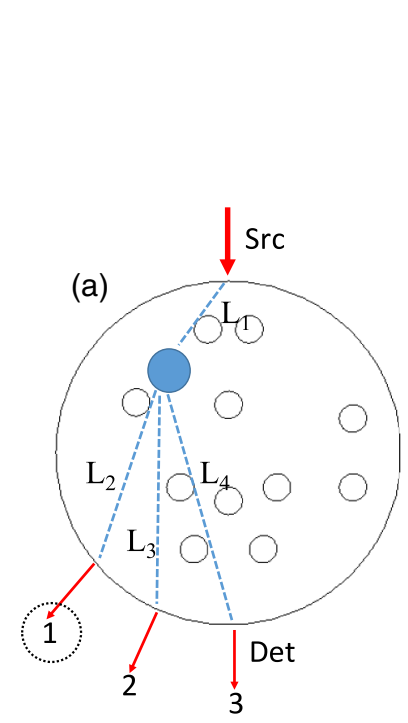

(b)

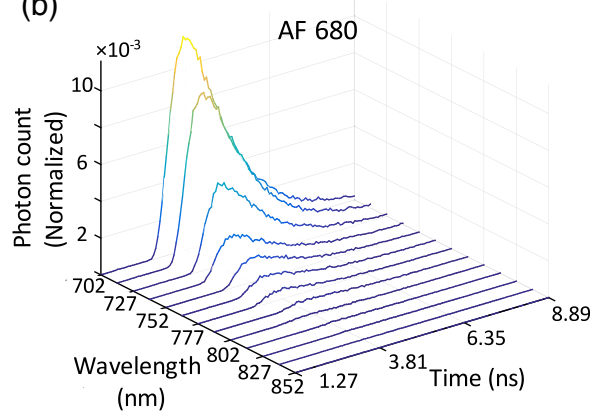

(d)

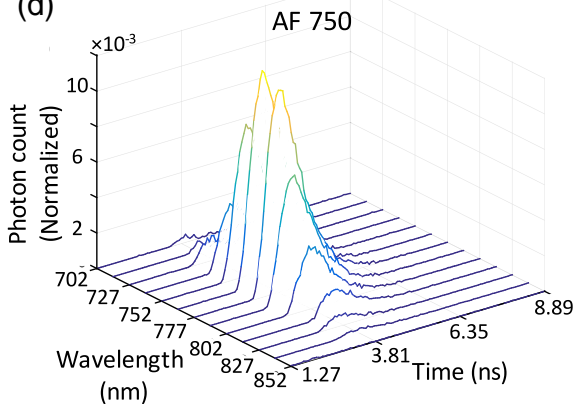

(c)

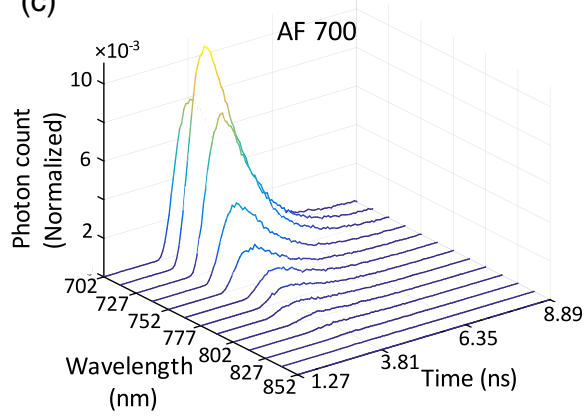

(e)

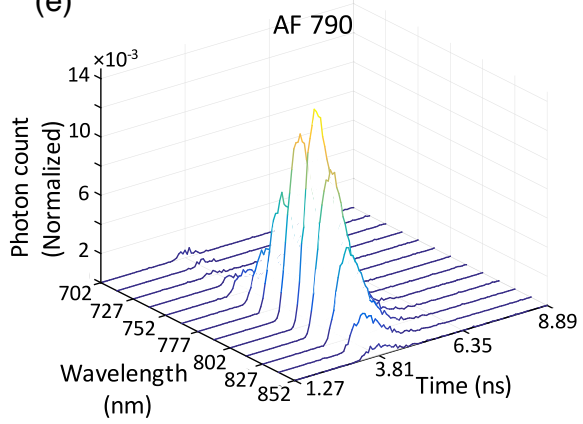

Fig. 4 (a) Geometry used for experimental measurement of the signature library with three source-detector pair combinations. Example measured signatures for (b) AF680, (c) AF00, (d) AF750, and (e) AF790, respectively. 
phantom base. By rotating the detector to three angles with labels 1,2 and 3, we can measure the temporal and spectral signatures corresponding to three different path lengths: $L_{1}+$ $L_{2}=23 \mathrm{~mm}, L_{1}+L_{3}=25 \mathrm{~mm}$, and $L_{1}+L_{4}=27 \mathrm{~mm}$. This process was repeated for all four fluorophores (AF680, AF700, AF750, and AF790), so that a library containing 12 signatures in total was generated. Example signatures are shown in Figs. 4(b)-4(e) for AF 680 [Fig. 4(b)], AF700 [Fig. 4(c)], AF750 [Fig. 4(d)], and AF790 [Fig. 4(e)], corresponding to $L=23 \mathrm{~mm}$. Here, each signature was normalized by the summation of all temporal-spectral photons, and would be transformed to a column vector in $\mathbf{S}$ defined in Eq. (2).

\subsubsection{Method 2: calculated signature library}

More generally it is desirable to be able to directly calculate (estimate) the library based on the photophysical properties of the fluorophores and mathematical models of light propagation in diffusive media. The latter requires knowledge of the optical properties of the diffusive media, in particular the absorption coefficient $\mu_{\mathrm{a}}$ and the reduced scattering coefficient $\mu_{\mathrm{s}}^{\prime}$. In general, these are unknown, so we used our instrument to make time-resolved transmission measurements to first determine the optical properties of the medium, as has been reported previously. ${ }^{30,31}$

For this, we used the broadband emission of our laser (path \# 2, Sec. 2.1) to illuminate the sample at the range of fluorescence emission wavelengths and measured the time-resolved spectral signal through the medium. We then fit the following timeresolved diffusion equation to data at each wavelength channel:

$T\left(\mathbf{r}_{s}, \mathbf{r}_{d}, t\right)=A \int_{t} \operatorname{TIRF}\left(t-t^{\prime}\right) G\left(\left|\mathbf{r}_{d}-\mathbf{r}_{s}\right|, t-t^{\prime}\right) \mathrm{d} t^{\prime}$,

where $\operatorname{TIRF}(t)$ is the temporal impulse response function of the system which could be measured experimentally. $G$ denotes the Green's function from the source to the detector, and has the form

$$
G=\frac{c}{(4 \pi D c t)^{3 / 2}} \exp \left(-\frac{\left|\mathbf{r}_{d}-\mathbf{r}_{s}\right|^{2}}{4 D c t}-\mu_{\mathrm{a}} c t\right),
$$
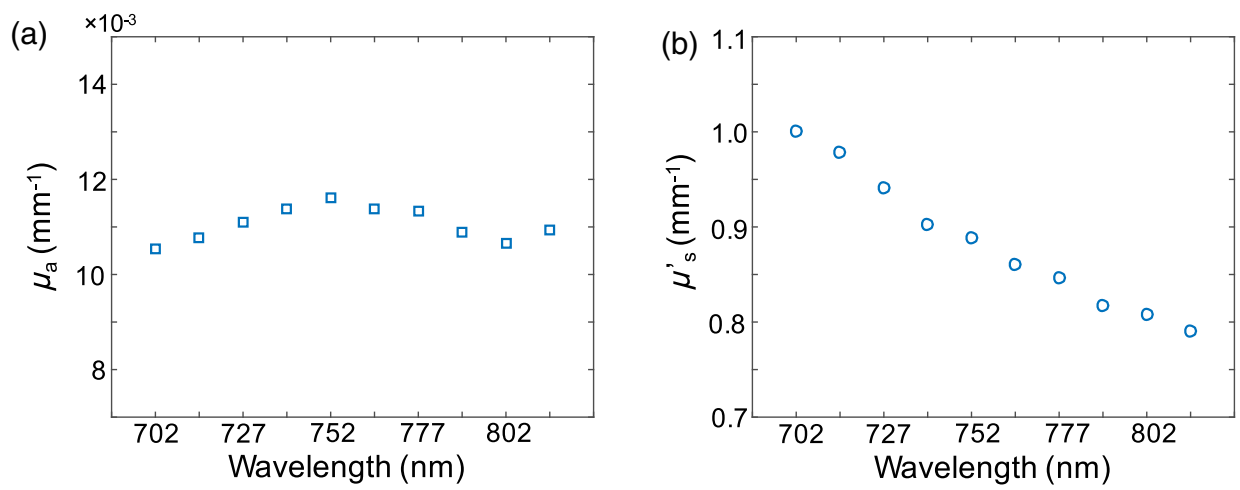

(c)

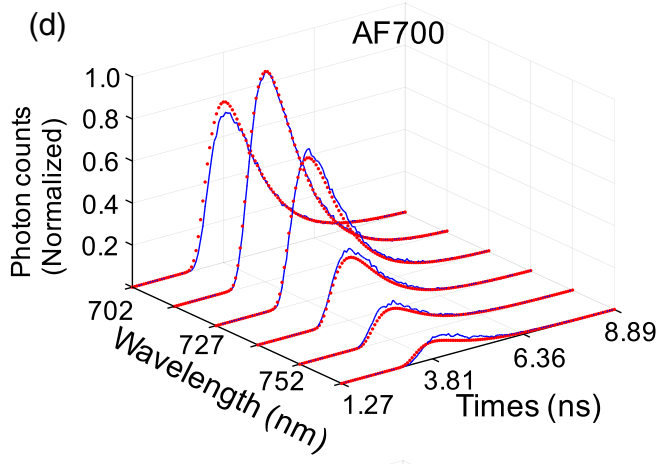

(e)
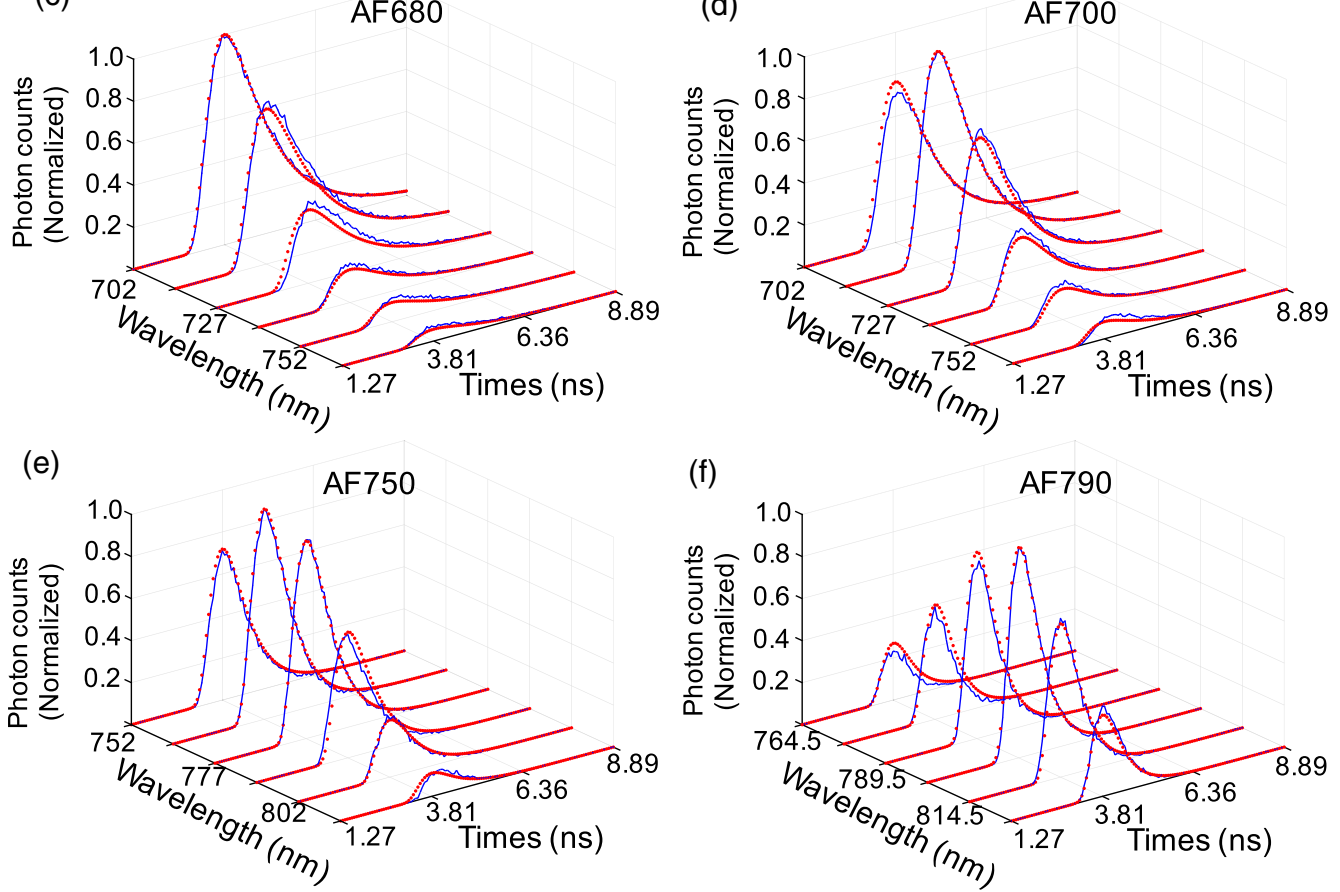

Fig. 5 (a) Measured wavelength-dependent absorption coefficient and (b) reduced scattering coefficient of the phantom. Experimentally measured (blue line) and computed fluorescence signatures (dotted red line) for (c) AF680, (d) AF700, (e) AF750, and (f) AF790, respectively. 

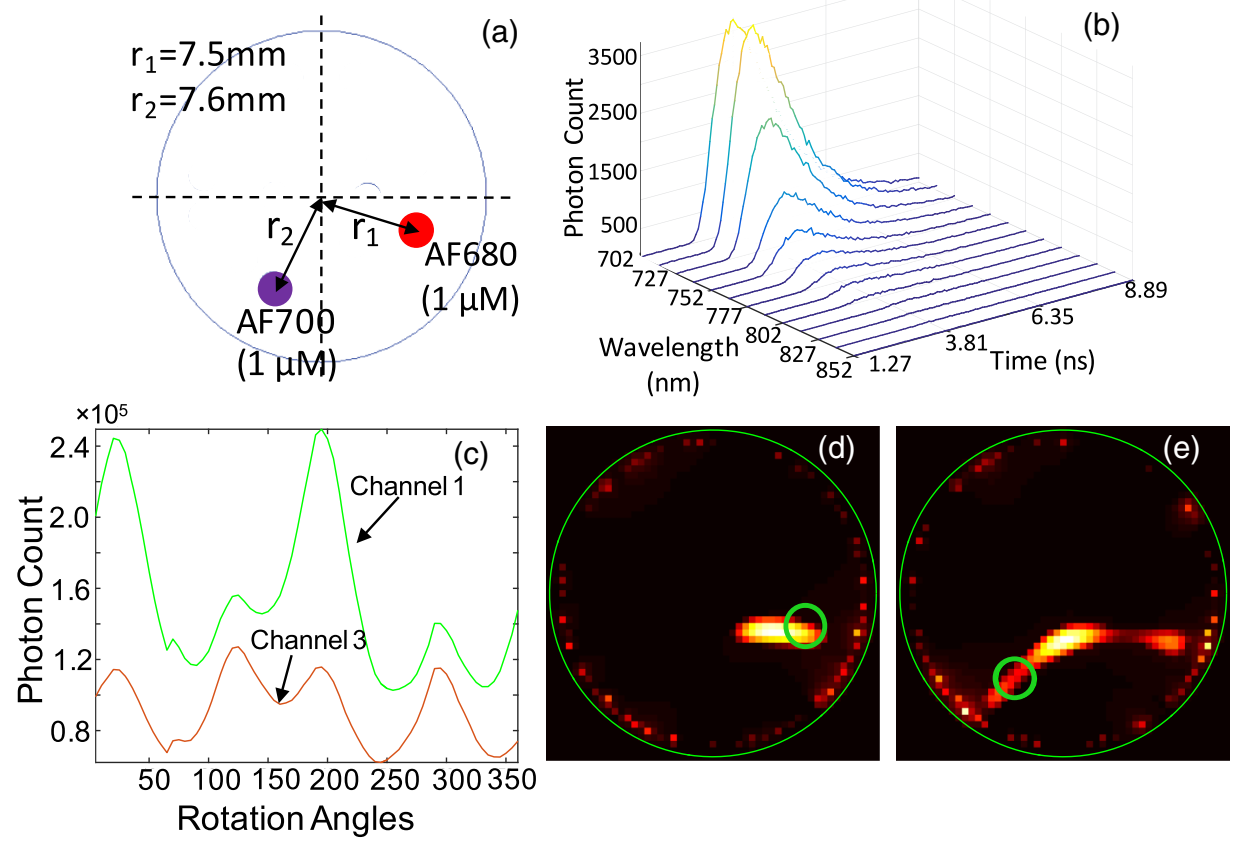

Fig. 6 (a) Placement of two fluorophores in the phantom. (b) Example measurement from a single source-detector projection. (c) Example measured CW traces corresponding to the first (green) and third (red) wavelength channels (centered at 702 and $727 \mathrm{~nm}$ ). Image reconstructions for the same data corresponding to (d) 702 and (e) $727 \mathrm{~nm}$, illustrating the significant bleed associated with pure spectral data.
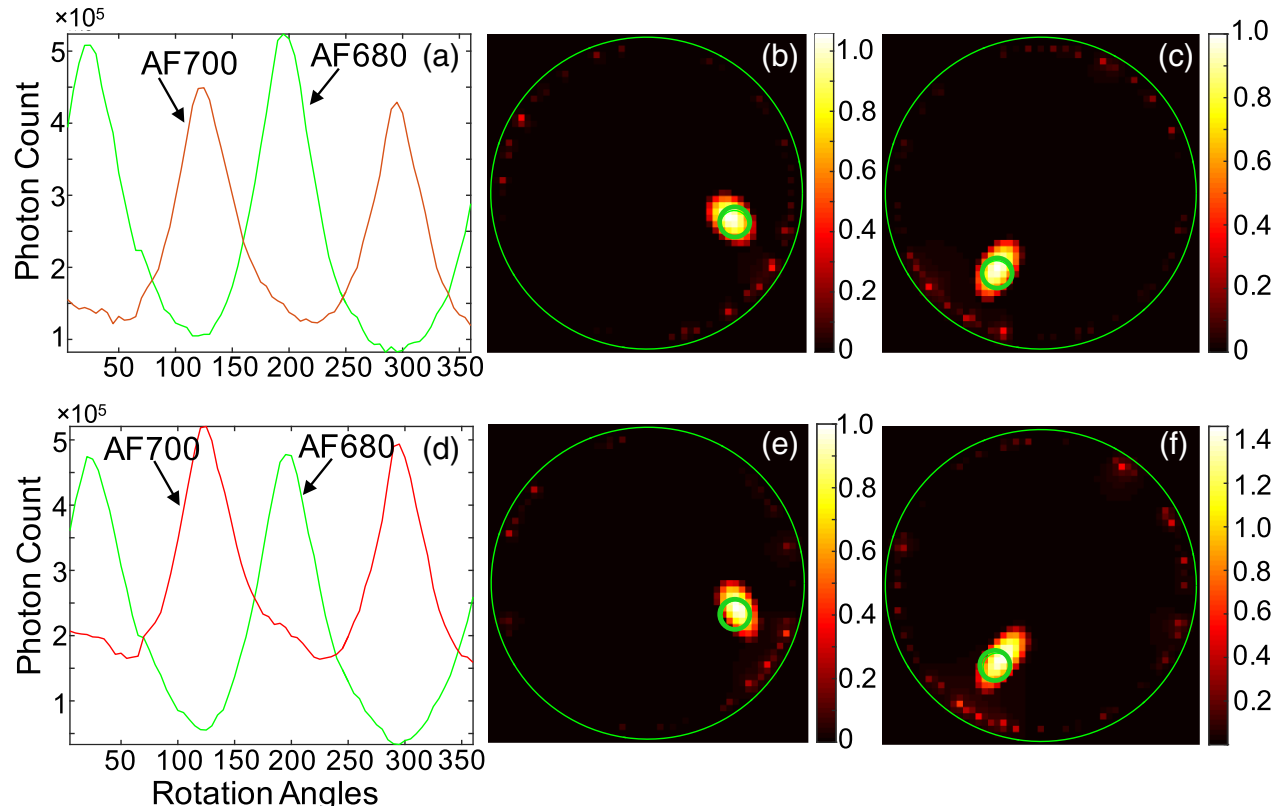

Fig. 7 (a) Demixed signal as a function of rotation angle for AF680 (green) and A700 (red) using the experimental signature library. The corresponding reconstructions for (b) AF680 and (c) AF700 are shown. (d) Example demixed fluorescent signals for AF680 (green) and AF700 (red) using the computed signature library, as well as the (e) AF680 and (f) AF700 reconstructions.

where $D=1 /\left[3\left(\mu_{\mathrm{a}}+\mu_{\mathrm{s}}^{\prime}\right)\right]$ and $c$ is the light velocity in the medium. Here, the parameter $A$ is a scalar constant that accounts for the system calibration, which depends on, e.g., the specific source power, detector efficiency. For curve fitting, we used the "Isqcurvefit" function in MATLAB ${ }^{\circledR}$.
This procedure retrieved $\mu_{\mathrm{a}}$ and $\mu_{\mathrm{s}}^{\prime}$ for each of the detection wavelength channels of our system. Example measured wavelength-dependent optical properties for our phantom are shown in Figs. 5(a) and 5(b), and these are generally in agreement with the literature values for intralipid in the NIR range. ${ }^{26}$ Using these 
data, we then computed the signature library for each fluorophore and pathlength combination using

$S\left(\mathbf{r}_{s}, \mathbf{r}_{d}, t\right)=\int_{t} F l\left(\mathbf{r}, t-t^{\prime}\right) W\left(\mathbf{r}_{s}, \mathbf{r}_{d}, \mathbf{r}, t^{\prime}\right) \mathrm{d} t^{\prime}$,

where $F l(\mathbf{r}, t)$ denotes the time-resolved emission light profile of the fluorophore, which incorporates quantum yield, lifetime information, as well as relative absorption and emission efficiency for the laser and wavelength combination. $W$ is the time-dependent weight function and has the form

$W\left(\mathbf{r}_{s}, \mathbf{r}_{d}, \mathbf{r}, t\right)=\int_{t} G^{x}\left(\mathbf{r}_{s}, \mathbf{r}, t^{\prime}\right) G^{m}\left(\mathbf{r}_{d}, \mathbf{r}, t-t^{\prime}\right) \mathrm{d} t^{\prime}$,

where $G^{x}$ and $G^{m}$ denote the excitation and emission Green's function, respectively. To find $F l(\mathbf{r}, t)$, we first measured the time-resolved fluorescence profile of each fluorophore in a nonscattering tube, which incorporated the relative emission at each wavelength and the fluorophore lifetime. We then convolved this with computed Green's functions for each pathlength combination (23, 25, and $27 \mathrm{~mm}$ as above). Example simulated signatures for AF680, AF700, AF750, and AF790 (red dotted line) are shown in Figs. 5(c)-5(f), along with the corresponding experimental signatures (blue line). We plotted six dominant wavelength channels for each fluorophore type. By inspection, the signatures generated by the two methods were very similar, and the computed cosine similarity between the simulated and experimental signatures was greater than $99 \%$ for all fluorophore types.

\section{Results and Discussion}

\subsection{Imaging Two Fluorophores Concurrently}

We first evaluated the performance of our instrument and algorithm in simultaneously imaging Alexa Fluor 680 (AF680) and AF700. AF680 and AF700 have emission peaks separated by only $20 \mathrm{~nm}$ and lifetimes separated by only $0.2 \mathrm{~ns}$ (1.2 and $1.0 \mathrm{~ns}$, respectively), and are therefore in general difficult to image simultaneously with either spectral or temporal data alone. The positions of the two fluorescent targets in this example are shown in Fig. 6(a). A representative measurement from a single source-detector pair is shown in Fig. 6(b). In total we collected 432 such measurements from a single axial slice. There are significant contributions from both fluorophores in the first three detector channels (centered at 702, 714.5, and $727 \mathrm{~nm}$, respectively). We plotted the data from the first and third channels as a function of rotation angle for one source-detector pair, along with the corresponding tomographic reconstructions [Figs. 6(d) and 6(e)] for these data. By inspection, significant cross-talk was observed between the channels, illustrating the challenges associated with imaging two closely overlapping fluorophores within the NIR wavelength range.

We next implemented our two-stage imaging algorithm ${ }^{23}$ and generated the extended library for demixing of the fluorophores (step 1) using either the experimental or computational methods. The demixed signals from the two fluorophores and the associated reconstruction for the experimental library are shown in Figs. 7(a)-7(c). Likewise, the demixed signals and reconstructions obtained using the computational method are shown in Figs. 7(d)-7(f).

Both methods for computing the library were able to demix the signal contributions from the two fluorophores. The image cross-talk between the two fluorophores was zero with the two methods, compared to $61.23 \%$ for the "naïve" approach shown in Fig. 6(e). In the final images, the targets were reconstructed with only $0.71,0.5,0.5$, and $1.12 \mathrm{~mm}$ center-to-center error from the known positions of the targets for the cases in Figs. 7(b), 7(c), 7(e), and 7(f) respectively. In combination, this illustrates the ability of the algorithm to separate and image two highly overlapping fluorophores with experimental data.

\subsection{Computed Error in the Demixing Stage}

We next studied the quantitative error introduced at the demixing stage as follows. We scanned the phantom with a tube of either AF680 or AF700 (but not both) present in the same position as above, and then summed them numerically in MATLAB ${ }^{\circledR}$. We then applied the demixing algorithm (stage 1)

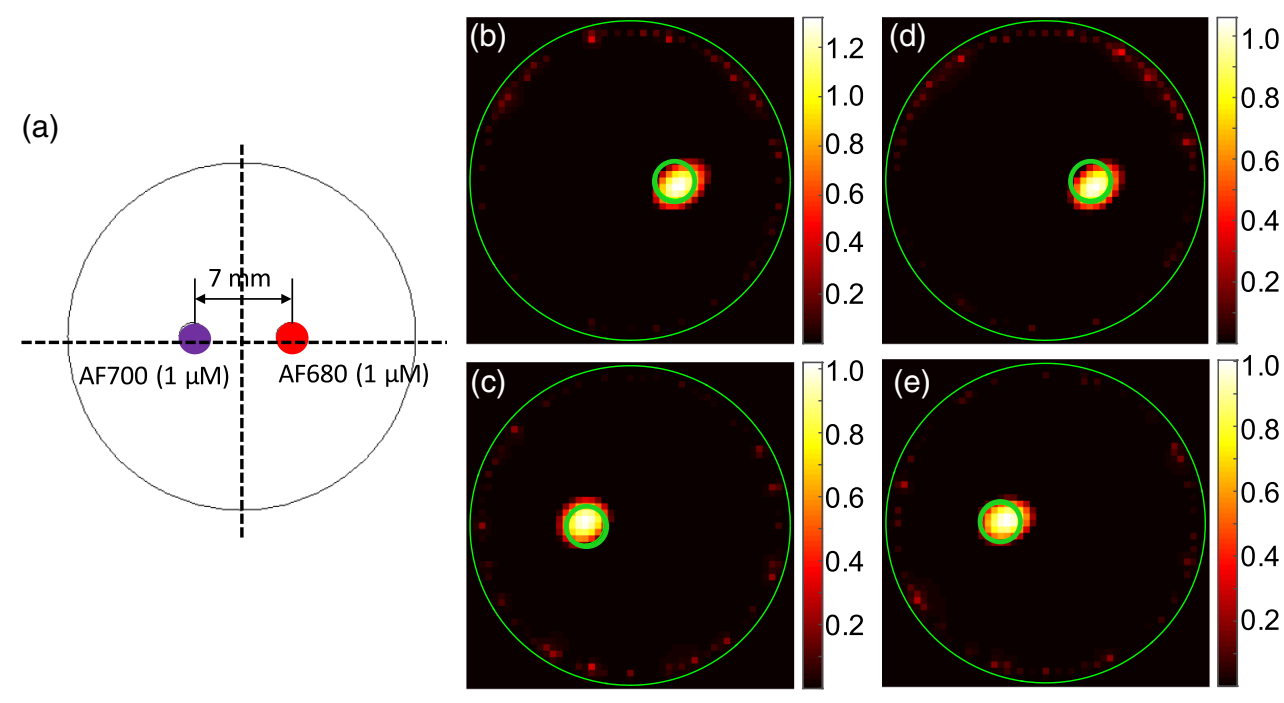

Fig. 8 (a) Placement of fluorophores at deep-seated position in the optical phantom. Reconstruction of (b) AF680 and (c) AF700 with the experimental library. Reconstruction of (d) AF680 and (e) AF700 with the computed library. 
and computed the point-by-point quantitative error from the original signals using

$e(f)=\frac{\left\|\hat{\mathbf{M}}_{f}-\mathbf{M}_{f}\right\|_{F}}{\left\|\mathbf{M}_{f}\right\|_{F}}$

where $\mathbf{M}_{f}$ is the ideal data for individual fluorophore, $\hat{\mathbf{M}}_{f}$ is the demixed data from the mixed signal, and $\|\cdot\|_{F}$ is the Frobenius norm. This error indicates the error associated with the demixing algorithm alone, primarily due to mismatches between the experimental data and the assumed extended fluorophore library. Errors were computed as $10.24 \%, 11.65 \%$ for the experimental library, and $18.91 \%, 23.93 \%$ for the computed library, for AF680 and AF700, respectively. Errors for the phantom where both targets were present [Figs. 7(a) and 7(d)] relative to the ideal data for individual fluorophores were slightly higher (a)
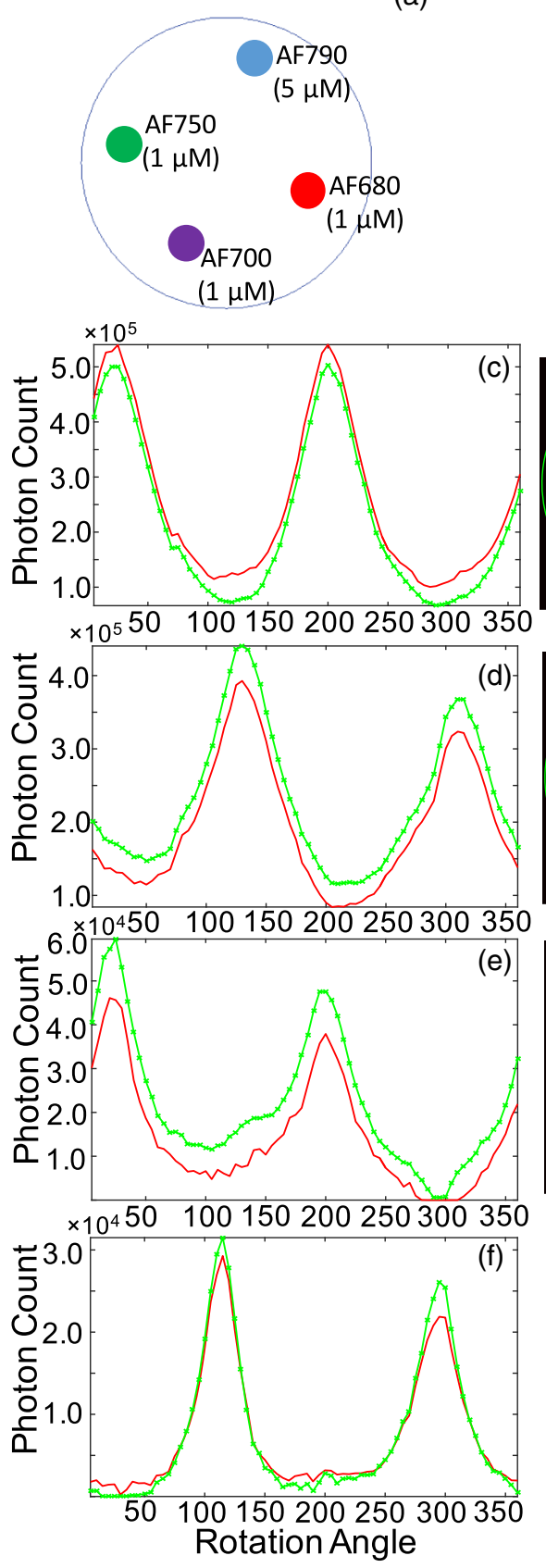

(b)

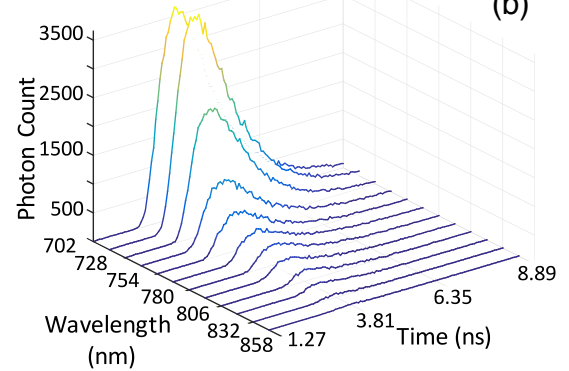

(nm)
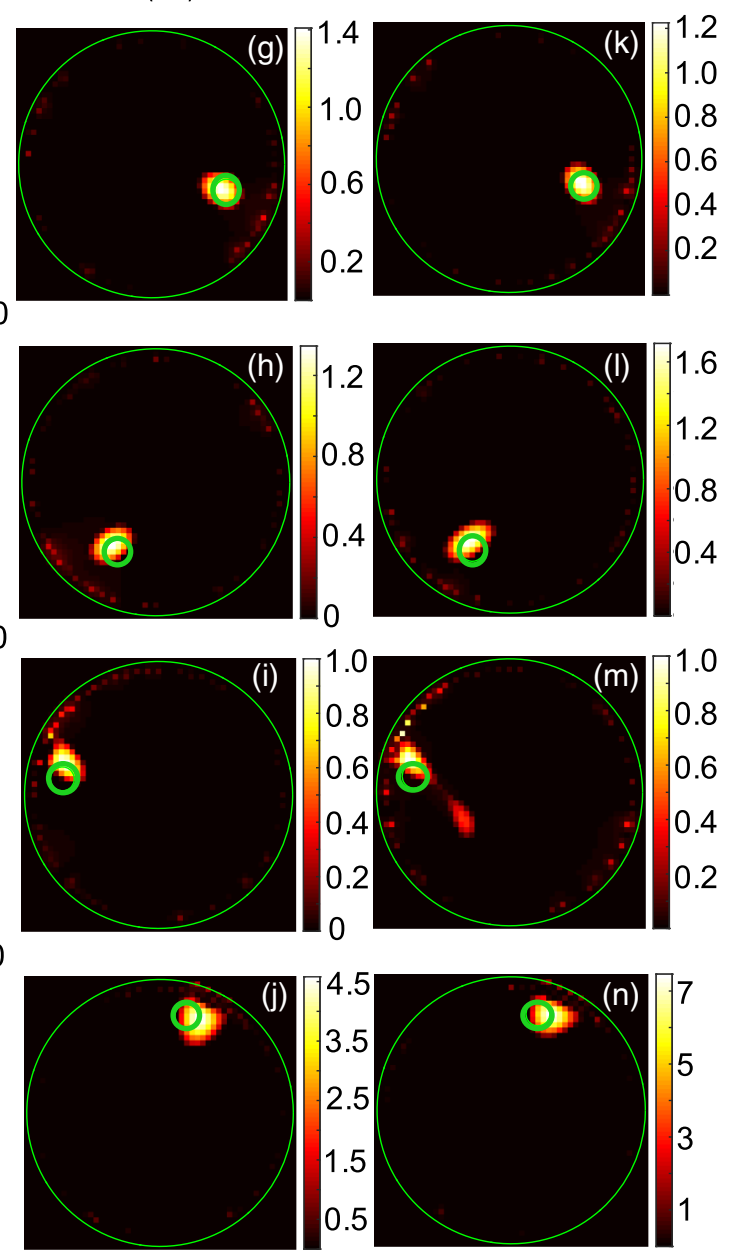

Fig. 9 (a) Placement of four fluorophores in the optical phantom. (b) Example measurement from a single source-detector projection. (c)-(f) Example CW traces after demixing with experimental (red solid) and computed (green $\mathrm{x}$ ) fluorescent signature libraries corresponding to AF680, AF700, AF750, and AF790, respectively. (g)-(j) Reconstructions of AF680, 700, 750, and 790 obtained using data demixed with experimentally measured fluorescence library, respectively. (k)-(n) Reconstructions of AF680, 700, 750 , and 790 obtained using data demixed with computed fluorescence signature library, respectively. 
(specifically, $16.07 \%, 14.54 \%$ and $24.45 \%, 25.71 \%$, respectively). We attribute this increased error to the changes in optical properties due to the second tube, which were not modeled in the extended libraries. Although the error is somewhat high, it is important to note that this is a point-by-point error, but the overall signal shape was reproduced quite well in all cases. Moreover (and as we have noted before ${ }^{23}$ ), the image reconstruction in the second stage of the algorithm was quite robust to small errors in signal estimation in the first stage, i.e., since zero cross-talk was observed, and the target positions were accurately reconstructed. The final relative reconstructed concentration ratio for AF680:AF700 was 1.05:1 and $1: 1.5$ for the experimentally measured and computed libraries, respectively (the true ratio was $1: 1)$.

In addition, we tested of our system in imaging multiple targets placed at positions deeper in the medium. AF680 and AF700 were positioned near the center of the phantom, and separated by 7-mm center-to-center as shown in Fig. 8(a). Analogous to the above, reconstructed images obtained using the experimental library are shown in Figs. 8(b) and 8(c), and the final relative reconstructed concentration ratio was $1.3: 1$. Likewise, the reconstructions for AF680 and AF700 obtained using the computed library are shown in Figs. 8(d) and 8(e), respectively. The final relative concentration ratio here was 1.06:1. The error in reconstruction of the position of the targets (centerto-center) was $0.5,0,0.71$, and $0.5 \mathrm{~mm}$ in Figs. 8(b)-8(e), respectively.

\subsection{Four-Fluorophore Phantom}

We then performed tomographic imaging of four fluorophores in our phantom model. The positions and concentrations of AF680, AF700, AF750, and AF790 are shown in Fig. 9(a). An example measured signal for a single source-detector pair is shown in Fig. 9(b). Red curves in Figs. 9(c)-9(f) show the demixed signal (after step 1) for each fluorophore where the experimentally measured library was used. The corresponding reconstructed images for each fluorophore are shown in Figs. 9(g)-9(j). As above, zero cross-talk was observed between fluorophores for each of the reconstructed images, indicating the ability of the algorithm to efficiently demix four fluorophores. The center-to-center error in the position of the reconstructed fluorophores was $0.5,0.71,1.58$, and $1 \mathrm{~mm}$ for each fluorophore, respectively. The relative fluorophore concentrations for AF680: AF700: AF750: AF790 was 1.4:1.3:1:4.6 which is in relatively good agreement with the true ratios of $1: 1: 1: 5$. We attribute most of this quantitative error to the impact of optical properties (absorption) of the fluorescent targets on the measured signals, which were not modeled in our approach. Our extended library could be expanded to incorporate this in the future.

Likewise, the green curves in Figs. 9(c)-9(f) show the demixed signal for each fluorophore with the computed library, and Figs. 9(k)-9(n) show the corresponding image reconstructions. Zero cross-talk was observed among fluorophores. The centerto-center position errors of the reconstructed targets were 0.5 , $0.71,1.58$, and $1 \mathrm{~mm}$, respectively. The final concentration ratio of the image reconstructions was about $1.2: 1.6: 1: 7$, which is in reasonable agreement with the true values, considering the complexity of the phantom model. We note that the error associated with the computed library was higher than that obtained with the experimentally measured library. This was expected, but nevertheless the computed library is practically preferable in many real experimental situations.

\subsection{Colocalized Fluorophores}

Last, we considered the potential impact of spatially overlapping (colocalized) fluorophores on the instrument and algorithm. In this configuration, we placed AF680 and AF750 at one location and AF700 and AF790 at a second location. The rationale here was that these combinations were most likely to produce unintended bleed among the different fluorophore channels. To avoid chemical interactions between different fluorophores, we designed a structure made with seven capillary tubes, six of which were filled with one of the two types of fluorophores. The configuration of this phantom is shown in Fig. 10(a). Figures 10(b)-10(e) show the final reconstructions for each

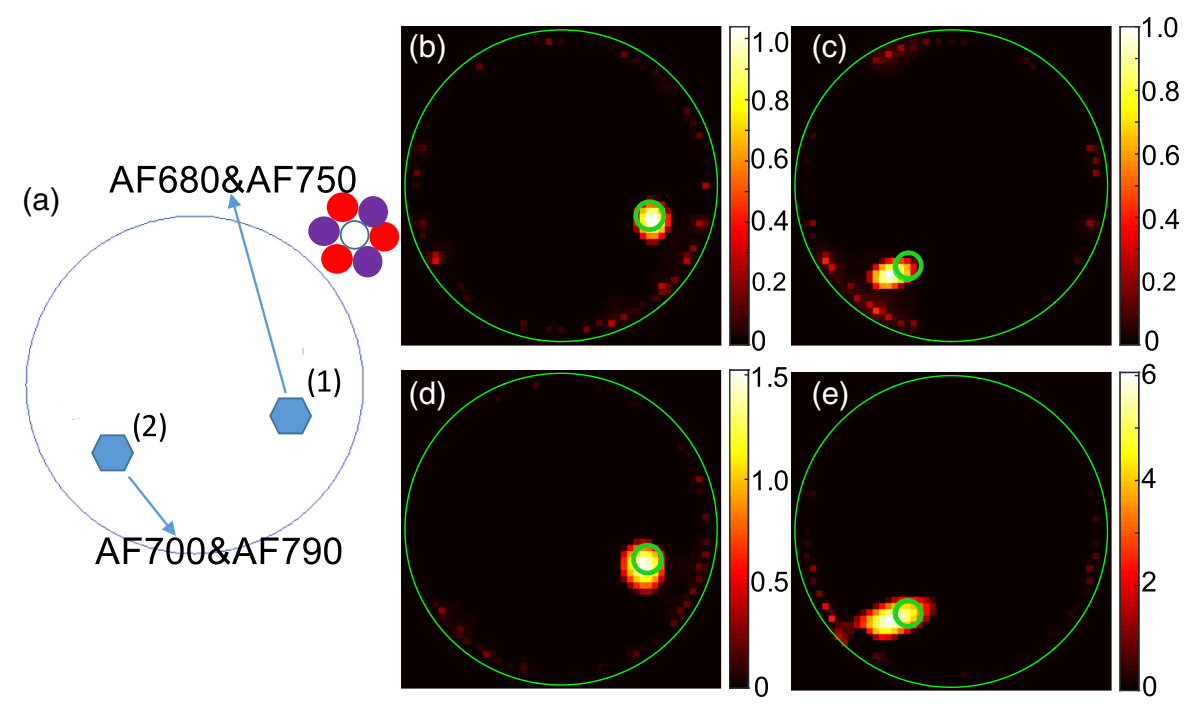

Fig. 10 (a) Placement of four fluorophores in the optical phantom, where AF680 and AF750, and AF700 and AF790 were arranged in pairs as shown. Image reconstructions for (b) AF680, (c) AF700, (d) AF750, and (e) AF790. 
fluorophore, where the demixing was performed with the experimentally measured library. Again, zero-cross talk was observed, illustrating the robustness of the algorithm with spatially overlapping targets. The center-to-center error in the position of the reconstructed flurophores was $0.71,0.5,0.71$, and $0.5 \mathrm{~mm}$, respectively. Last, the computed concentration ratios were $1.04: 1: 1.52: 6.07$, which is in relatively good agreement with the true ratios of $1: 1: 1: 5$.

\section{Conclusion}

In summary, we developed a new FMT scanner with extended multiplexed imaging capabilities in the NIR range, and validated its performance with tissue-mimicking phantoms with fluorescent inclusions. The scanner was capable of acquiring both spectral and temporal data, which we analyzed using our previously developed algorithm, which sequentially demixes and then reconstructs the contributions from each fluorophore. ${ }^{23}$ In addition, we used the same instrument to measure the time-resolved transmittance through the sample in the NIR region, and fit these data to time-resolved diffusion theory to estimate the optical properties of the medium. Combined with knowledge of the fluorescence spectra and lifetime, this allowed us to compute (as opposed to experimentally measure) the extended signature library for the demixing step.

We showed experimentally that it was able to separate the emission signals from at least four NIR fluorophores with closely overlapping emission spectra and lifetimes using measured or computed extended libraries. Zero cross-talk was observed in the final reconstructed images in all cases, and the error in the reconstructed position was on the order of $1 \mathrm{~mm}$, indicating the robustness of the algorithm. The demixing error in the fluorophore signal was no more than $25 \%$ compared to pure signals when computed on a point-by-point basis. However, we note that the overall signal was qualitatively well reproduced, accounting for the accuracy of the final images. These demixing errors were primarily due to unmodeled sources of model-data mismatch, including absorption of fluorescent emission by other targets. These could be incorporated in our algorithm in the future to improve this error. As expected, the computed extended library yielded slightly larger errors than the measured library, but is in general more useful since it is difficult or infeasible to experimentally measure the extended library, e.g., in a mouse.

In this paper, we have shown some illustrative configurations of fluorophores, i.e., multiple targets at various depths, separations, and overlap. However, there are of course many other potential combinations, many of which we have tested but have not shown herein. We note that a major strength of our approach is the ability to decouple the demixing and reconstruction stages, so that the reconstruction stage could be further optimized in the future. For example, rather than use the diffusion approximation, we could use Monte Carlo or finite element modeling to compute the Jacobians for irregular or arbitrary tissue volumes. Overall, our approach (and that of others ${ }^{18-22}$ ) indicates that joint use of spectral and temporal data in the demixing stage significantly improves the multiplexing capabilities of FMT. Analogous to fluorescence microscopy, this could enable tomographic imaging experiments with multiple fluorescent reporters simultaneously probing different molecular targets in vivo.

\section{Acknowledgments}

This work was funded by a grant from the National Institutes of Health (R01EB012117).

\section{References}

1. V. Ntziachristos, C. Bremer, and R. Weissleder, "Fluorescence imaging with near-infrared light: new technological advances that enable in vivo molecular imaging," Eur. Radiol. 13(1), 195-208 (2003).

2. R. B. Schulz, J. Ripoll, and V. Ntziachristos, "Experimental fluorescence tomography of tissues with noncontact measurements," IEEE Trans. Med. Imaging 23(4), 492-500 (2004).

3. W.-T. Chen et al., "Arthritis imaging using a near-infrared fluorescence folate-targeted probe," Arthritis Res. Ther. 7(2), R310 (2005).

4. A. Petrovsky et al., "Near-infrared fluorescent imaging of tumor apoptosis," Cancer Res. 63(8), 1936-1942 (2003).

5. D. Citrin et al., "In vivo tumor imaging in mice with near-infrared labeled endostatin," Mol. Cancer Ther. 3(4), 481-488 (2004).

6. M. Funovics, R. Weissleder, and C.-H. Tung, "Protease sensors for bioimaging," Anal. Bioanal. Chem. 377(6), 956-963 (2003).

7. V. Ntziachristos et al., "Fluorescence molecular tomography resolves protease activity in vivo," Nat. Med. 8(7), 757-761 (2002).

8. R. Weissleder et al., "In vivo imaging of tumors with protease-activated near-infrared fluorescent probes," Nat. Biotechnol. 17(4), 375-378 (1999).

9. N.-H. Ho, R. Weissleder, and C.-H. Tung, "Development of watersoluble far-red fluorogenic dyes for enzyme sensing," Tetrahedron 62(4), 578-585 (2006).

10. X. Montet et al., "Tomographic fluorescence mapping of tumor targets," Cancer Res. 65(14), 6330-6336 (2005).

11. S. L. Gibbs-Strauss et al., "Noninvasive measurement of aminolevulinic acid-induced protoporphyrin ix fluorescence allowing detection of murine glioma in vivo," J. Biomed. Opt. 14(1), 014007 (2009).

12. V. Ntziachristos et al., "Visualization of antitumor treatment by means of fluorescence molecular tomography with an annexin v-cy5. 5 conjugate," Proc. Natl. Acad. Sci. U. S. A. 101(33), 12294-12299 (2004).

13. E. A. Schellenberger et al., "Optical imaging of apoptosis as a biomarker of tumor response to chemotherapy," Neoplasia 5(3), 187-192 (2003).

14. M. Dickinson et al., "Multi-spectral imaging and linear unmixing add a whole new dimension to laser scanning fluorescence microscopy," Biotechniques 31, 1272-1274 (2002).

15. Y. Hiraoka, T. Shimi, and T. Haraguchi, "Multispectral imaging fluorescence microscopy for living cells," Cell Struct. Funct. 27(5), 367-374 (2002).

16. H. Kobayashi et al., "Simultaneous multicolor imaging of five different lymphatic basins using quantum dots," Nano Lett. 7(6), 1711-1716 (2007).

17. F. Leblond et al., "Pre-clinical whole-body fluorescence imaging: review of instruments, methods and applications," J. Photochem. Photobiol. B 98(1), 77-94 (2010).

18. A. T. Kumar et al., "Fluorescence-lifetime-based tomography for turbid media," Opt. Lett. 30(24), 3347-3349 (2005).

19. W. L. Rice and A. T. Kumar, "Preclinical whole body time domain fluorescence lifetime multiplexing of fluorescent proteins," J. Biomed. Opt. 19(4), 046005 (2014).

20. W. L. Rice et al., "In vivo tomographic imaging of deep-seated cancer using fluorescence lifetime contrast," Cancer Res. 75(7), 1236-1243 (2015).

21. J. Chen, V. Venugopal, and X. Intes, "Monte Carlo based method for fluorescence tomographic imaging with lifetime multiplexing using time gates," Biomed. Opt. Express 2(4), 871-886 (2011).

22. Q. Pian et al., "Hyperspectral time-resolved wide-field fluorescence molecular tomography based on structured light and single-pixel detection," Opt. Lett. 40(3), 431-434 (2015).

23. V. Pera, D. H. Brooks, and M. Niedre, "Multiplexed fluorescence tomography with spectral and temporal data: demixing with intrinsic regularization," Biomed. Opt. Express 7(1), 111-131 (2016).

24. N. Valim, "Instrumentation and methods for time-resolved diffuse fluorescence imaging," PhD Thesis, Northeastern University (2014). 
25. Y. Mu and M. Niedre, "Fast single photon avalanche photodiode-based time-resolved diffuse optical tomography scanner," Biomed. Opt. Express 6(9), 3596-3609 (2015).

26. R. Michels, F. Foschum, and A. Kienle, "Optical properties of fat emulsions," Opt. Express 16(8), 5907-5925 (2008).

27. H. J. Van Staveren et al., "Light scattering in Intralipid-10\% in the wavelength range of 400-1100 nm," Appl. Opt. 30(31), 4507-4514 (1991).

28. Thermo Fisher Scientific, http://www.lifetechnologies.com/us/en/home/ life-science.html.

29. P. C. Hansen and M. Saxild-Hansen, "Air tools-a MATLAB package of algebraic iterative reconstruction methods," J. Comput. Appl. Math. 236(8), 2167-2178 (2012).

30. M. S. Patterson, B. Chance, and B. C. Wilson, "Time resolved reflectance and transmittance for the noninvasive measurement of tissue optical properties," Appl. Opt. 28(12), 2331-2336 (1989).
31. M. J. Niedre, G. M. Turner, and V. Ntziachristos, "Time-resolved imaging of optical coefficients through murine chest cavities," J. Biomed. Opt. 11(6), 064017 (2006).

Ying Mu is a postdoctoral fellow in the Department of Electrical and Computer Engineering at Northeastern University. She received her PhD from Northeastern University in August 2016.

Vivian Pera is a postdoctoral fellow in the Department of Biomedical Engineering at Boston University. She received her PhD from Northeastern University in 2015.

Mark Niedre is an associate professor of electrical and computer engineering and bioengineering at Northeastern University. He received his $\mathrm{PhD}$ degree from the University of Toronto in the Department of Medical Physics in 2004. 